\title{
The pFFT accelerated BEM for the simulation of 3-D acoustic problems
}

\author{
Z. Y. Yan ${ }^{1,2}$ \& C. Z. $\mathrm{Li}^{1}$ \\ ${ }^{1}$ Department of Aerodynamics, \\ Nanjing University of Aeronautics and Astronautics, P. R. China \\ ${ }^{2}$ State Key Laboratory of Structural Analysis for Industrial Equipment, \\ Dalian University of Technology, P. R. China
}

\begin{abstract}
The precorrected-FFT acceleration technique is successfully applied in the boundary element method for the simulation of 3-D acoustic radiation problems. The constant triangular element is employed in the simulation. The computational cost, the consumed memory and the accuracy of the current method are demonstrated and analyzed through the simulation of the acoustic radiation from a pulsating sphere. Both the surface and the exterior field dimensionless acoustic pressures are presented to show the precision of this method.
\end{abstract}

Keywords: BEM, pFFT, acoustic radiation.

\section{Introduction}

Boundary element method (BEM) [1] is a very popular numerical approach. The origin of this method can be traced to 1960s. Compared with other numerical methods, such as the finite element method and the finite difference method, it has the advantages that mesh need only to be generated on the boundary and the boundary conditions at infinity can be automatically satisfied. Therefore, it is very suitable to simulate the exterior acoustic problems.

It is well-known that the final influence matrix of the conventional BEM is fully-populated. As a result, both the computational cost and the consumed memory are very expensive to solve large-scale problems by using the conventional BEM. To overcome these difficulties, some acceleration techniques were developed in the past decades. The BEM accelerated by the fast multipole 
expansion method (FMM) [2-5] had been developed and applied to nearly all the fields that the conventional BEM can be employed. The BEM accelerated by the precorrected fast Fourier transform method (pFFT) [6] was widely applied as well [7-12]. In most cases, the constant boundary element was used in the pFFTBEM. Recently, two pFFT-BEMs with higher order boundary element $[13,14]$ were presented for the simulation of wave-body interactions. The pFFT algorithm is quite independent of the Green functions, even for highly oscillatory kernels $[15,16]$. Therefore, the pFFT-BEM is very suitable for the simulation of wave propagation problems. Usually, the iterative solver based on the generalized minimal residual algorithm (GMRES) [17] is used together with these acceleration techniques to form the fast BEMs. Lately, the adaptive cross approximation (ACA) method [18] gets more and more attention and applications due to its easy implementation.

To implement the BEM, the computation of the weakly singular integrals, the strongly singular integrals and the hypersingular integrals [19-24] appearing in the boundary integral equations is indispensable. To date, a lot of research has been done on the computation of such kinds of singular integrals, especially the hypersingular integrals.

The BEM based on the Helmholtz integral equation (HIE) has long been employed to simulate acoustic problems [25-27]. In this paper, the development of the pFFT-BEM for the 3-D exterior acoustic radiation problems based on the HIE is presented. The motivation for such a development is due to the fact that the pFFT acceleration technique is relatively kernel independent and it is not very difficult to implement it based on the previous code for elastostatics [9], elastodynamics [15] and acoustic scattering [10, 12]. As usual, constant boundary element is applied in all the simulation.

This paper is organized as follows. Firstly, a brief introduction about BEM and its related topics is presented. Then, the pFFT-BEM for exterior acoustic radiation problems based on the HIE is developed. Next, an example of the pulsating sphere radiation is presented to validate the developed method and the code. The computational cost, consumed memory and accuracy of the pFFTBEM for acoustic radiation problems based on the HIE are analyzed and demonstrated. Lastly, some conclusions are drawn.

\section{The pFFT-BEM for acoustic radiation problems}

\subsection{Boundary integral formulation for acoustic radiation problems}

The governing equation about the acoustic pressure $\varphi$ for the steady-state linear acoustics in the frequency domain is the well-known Helmholtz equation [21]

$$
\left(\nabla^{2}+k^{2}\right) \varphi=0
$$

where $k$ is the wave number. 


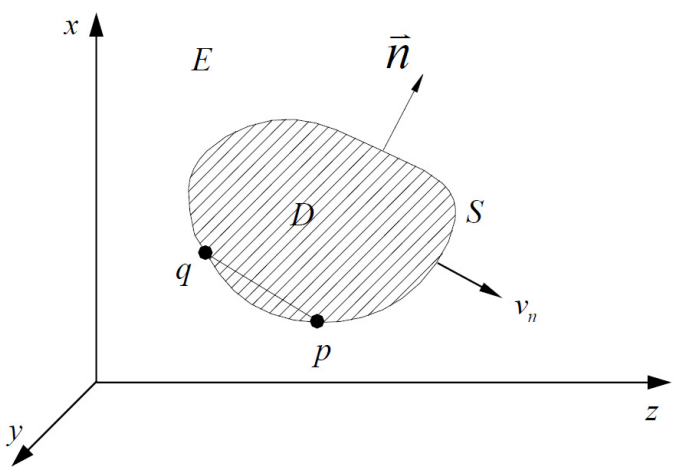

Figure 1: $\quad$ Illustration of a vibrating structure.

Consider the 3-D acoustic radiation problem from a closed vibrating surface $S$ as shown in Figure 1. For this exterior problem, $\vec{n}$ represents the inward unit normal vector. The symbol $E$ represents the exterior domain and the symbol $D$ represents the interior domain. $p$ and $q$ are respectively the source point and the field point.

The Neumann boundary condition [21] on the surface $S$ can be expressed as

$$
\frac{\partial \varphi}{\partial n_{q}}=i \omega \rho v_{n}
$$

where $\omega$ represents the circular frequency; $\rho$ represents the density of the acoustic medium and $v_{n}$ denotes the normal velocity on the boundary.

Combining the Sommerfeld radiation condition [21, 28], an equivalent Helmholtz boundary integral formulation (HIE) about the acoustic pressure $\varphi$ can be derived from eqn (1) by using the Green's second identity as

$$
-c(p) \varphi(p)=\iint_{S}\left[G_{k}(p, q) \frac{\partial \varphi(q)}{\partial n_{q}}\right] d S_{q}-\iint_{S}\left[\frac{\partial G_{k}(p, q)}{\partial n_{q}} \varphi(q)\right] d S_{q},
$$

where the 3-D free-space Green's function $G_{k}$ for the steady state linear acoustic problems is given by

$$
G_{k}(p, q)=\frac{1}{4 \pi} \frac{e^{-i k r}}{r}, r=|p-q| .
$$

In this equation, $i$ represents the imaginary unit and $r$ is the Euclidean distance between the source point $p$ and the field point $q$. Since the triangular constant boundary element is applied in the code, the solid angle $c(p)$ at the centroid of an element equals to 0.5 .

In the integral operator notations $[21,28]$, eqn (3) can be expressed as

$$
\left[-\frac{1}{2} I+M_{k}\right][\varphi]=L_{k}\left[\frac{\partial \varphi}{\partial n}\right]
$$


where $I$ represents the identity integral operator. For an arbitrary function $\mu$, the integral operators $L_{k}, M_{k}[28]$ are defined as

$$
\begin{gathered}
L_{k}[\mu]=\iint_{S}\left[G_{k}(p, q) \mu(q)\right] d S_{q}, \\
M_{k}[\mu]=\iint_{S}\left[\frac{\partial G_{k}(p, q)}{\partial n_{q}} \mu(q)\right] d S_{q} .
\end{gathered}
$$

\subsection{The precorrected-FFT algorithm}

If the integrand of a boundary integral can be expressed as a function of the form [12]

$$
f\left(r(p, q), r_{i}(p, q)\right) \times Q(q),
$$

then it can be computed by using the pFFT technique.

In the pFFT technique, a three-dimensional problem is firstly discretized into $n$ surface elements. Then a rectangular parallelepiped is constructed to enclose it. It is subdivided into an array of small cubes so that each small cube contains only a few elements. The number of cubes is determined based on the balance between the accuracy and the efficiency. Based on these cubes, the near-field and far-field of each element can be determined. The near field of an element in one cube is composed of all the elements in the cubes just close to this cube and the far field is formed by all the other elements. Boundary integrals in the near-field are computed directly using the Gaussian quadrature. A $3 \times 3 \times 3$ array of grid points is uniformly distributed on each cube. Then the source on an element is projected onto the grid points on the cube that containing this element. The projection is based on a polynomial interpolation. On these uniformly distributed grid points, the boundary integral has a piecewise-smooth discrete convolution form. It can be computed approximately using the discrete fast Fourier transform technique. After that, the boundary integral computed on the grid points is projected back onto the elements. In order to keep the accuracy, the near-filed interactions computed by this approximate method are removed and those computed by the direct method are added. Thus, the main steps $[9,12]$ in the pFFT-BEM can be summarized as:

(1) Discretization of the problem;

(2) Construction and superposition of a 3-D uniform grid on the discretized problem;

(3) Determination of the near- and far-field for each panel;

(4) Computation of the near-field interactions using the direct calculation;

(5) Projection of the sources onto the surrounding grid points based on a polynomial interpolation;

(6) Calculation of the grid-to-grid interactions using the discrete Fast Fourier Transformation;

(7) Projection of the grid-to-grid interactions back to elements; 
(8) Subtraction of the near-field interactions by the approximate method and summation of the near-field interactions by the direct calculation and farfield contributions by the approximate method;

(9) Finding the solutions using the iterative solver GMRES.

\subsection{Implementation of the HIE with the pFFT technique}

The near-field interactions are directly calculated using the Gaussian quadrature with $3 \times 3$ Gaussian points. A standard degenerate mapping or polar coordinate transformation is applied to eliminate the weakly singularity [29].

In the implementation of the pFFT algorithm, a key step is the calculation of the grid-to-grid interactions. In order to use the FFT technique, the grid-to-grid interactions should possess a discrete convolution form.

In eqn (5), the integrand of the boundary integral $L_{k}[\partial \varphi / \partial n]$ does satisfy the form (6), therefore this boundary integral can be calculated using the pFFT algorithm directly. However, the integrand of the boundary integral $M_{k}[\varphi]$ does not satisfy the form (6). It can be rewritten as $M_{k i}\left[n_{i} \varphi\right],(i=1,2,3)[10,12]$

$$
M_{k i} \mu=\iint_{S}\left[\frac{\partial G_{k}(p, q)}{\partial r} \frac{r_{i}}{r} \mu(q)\right] d S_{q} .
$$

Since the integrand of the boundary integral $M_{k i}\left[n_{i} \varphi\right]$ satisfies the form (6), each of these three boundary integrals can be calculated using the pFFT algorithm.

The main difference of the pFFT accelerated HIE for acoustic radiation and that for the acoustic scattering is the computation of the term related to the boundary conditions.

\section{Numerical examples}

To validate the fast BEM based on the pFFT accelerated Helmholtz integral equation (HIE), an example of the pulsating sphere acoustic radiation is simulated. In the simulation, the iterative tolerance of the GMRES iterative method is set as $10^{-4}$. It will affect the computational cost and the consumed memory. A $3 \times 3$ Gaussian quadrature is applied in the computation of the nearfield interactions. The simulation is run in a Linux system on a PC with $2.66 \mathrm{GHz}$ of the Intel(R) Core(TM)2 Quad CPU and with $8 \mathrm{~GB}$ of the physical memory.

Consider acoustic radiation from a sphere of radius $a=1 \mathrm{~m}$ with a uniform radial velocity $v_{0}$. The analytical solution [21] of the acoustic pressure $\varphi(r)$ is given by

$$
\frac{\varphi(r)}{\rho c v_{0}}=\frac{a}{r} \frac{i k a}{1+i k a} e^{-i k(r-a)}
$$




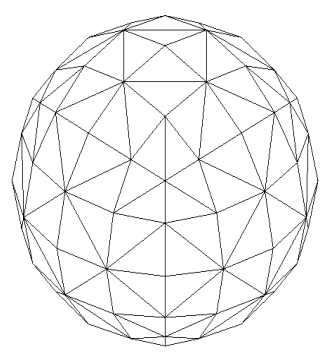

(a) 192 elements

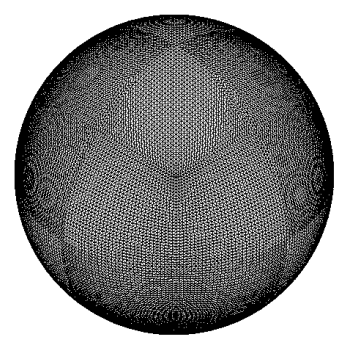

(b) 49664 elements

Figure 2: $\quad$ The sphere discretized by constant triangular surface elements.

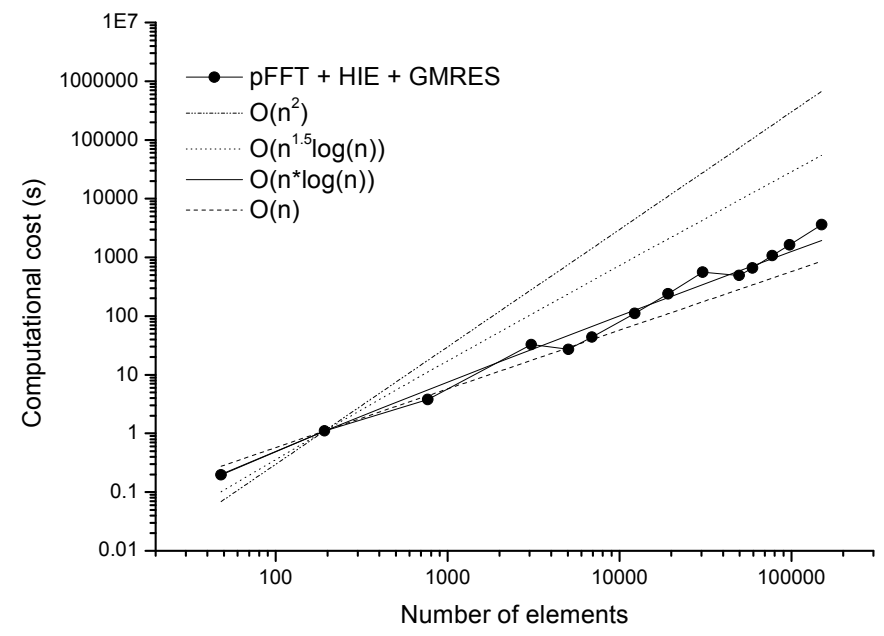

Figure 3: The computational cost as a function of the number of elements.

To examine the efficiency and the required memory of the pFFT-BEM based on the HIE, simulation is conducted for fourteen discretizations starting from the coarse mesh with 48 triangular elements to the finest mesh with 150338 triangular elements at the reduced frequency $k a=1.50294$. For simplicity, only the discretizations with 192 and 49664 triangular elements are displayed in Figure 2. Except the case with 48 elements for which the solutions converge at the iteration step 2, all the other cases converge at the iteration step 4 . The computational cost and the consumed memory as functions of the number of elements are respectively shown in Figures 3 and 4. For problem domains with small surface-to-volume ratios, the optimal order of the pFFT-BEM $O(n \log n)$ 
for the computational cost and $O(n)$ for the consumed memory $[6,11]$ can be achieved. For other problems, the computational cost of the pFFT-BEM is between $O(n \log n)$ and $O\left(n^{1.5} \log n\right)$ [11]. Therefore, the lines corresponding to $O\left(n^{2}\right), O\left(n^{1.5} \log n\right), O(n \log n)$ and $O(n)$ are also plotted in these two figures for reference. Because the simulation with the coarse mesh of 48 triangular elements is actually calculated by the direct method in the pFFT-BEM, all these lines are shifted to start from the point of 192 triangular elements. The same as those shown in the cases of acoustic scatterings [12], the computational costs of the pFFT-BEM based on the HIE oscillates along the line $O(n \log n)$ slightly and they seem to piecewisely parallel to the lines $O\left(n^{2}\right)$ or $O\left(n^{1.5} \log n\right)$. Figure 4 shows that the consumed memory oscillates along the line $O(n)$ slightly.

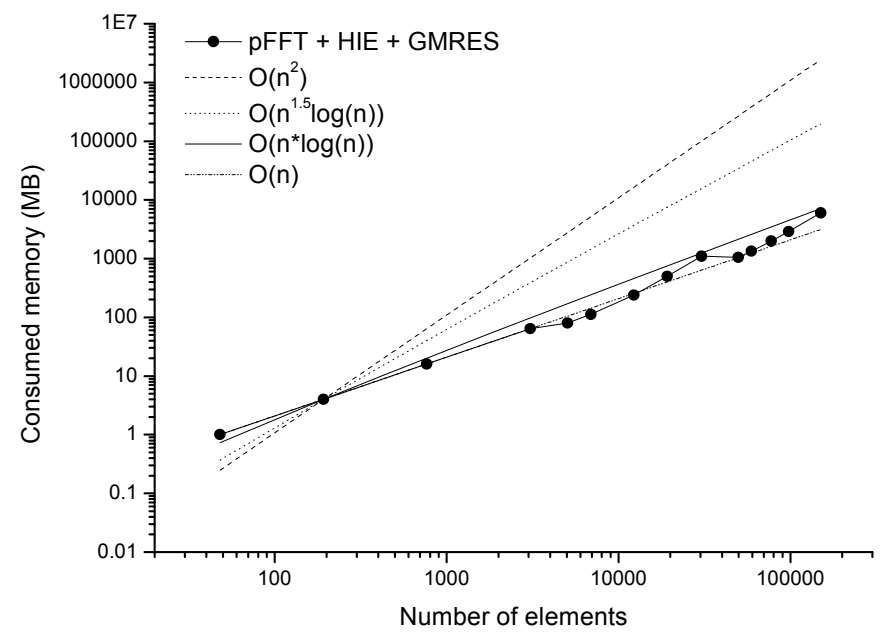

Figure 4: The consumed memory as a function of the number of elements.

To validate the code and show that accuracy of the pFFT-BEM, comparison between the dimensionless surface acoustic pressures on the circle $x=0$ as $k a=1.50294$ computed by the pFFT accelerated Helmholtz integral equation (HIE) and the corresponding analytical solutions is presented in Figure 5. Two discretizations with 19520 and 1503384 triangular elements are employed. The $L_{2}$-norm errors between the numerical solutions found by the pFFT accelerated HIE and the corresponding analytical solutions are respectively $1.84 \%$ and $1.16 \%$ for the discretizations with 19520 and 150338 elements. Although these errors are not too large, they are not as small as those we expected for the simulation with so many elements. This should be due to the fact that a nonuniqueness frequency exists near the reduced frequency $k a=0$ [21] for the boundary element method based on HIE. 


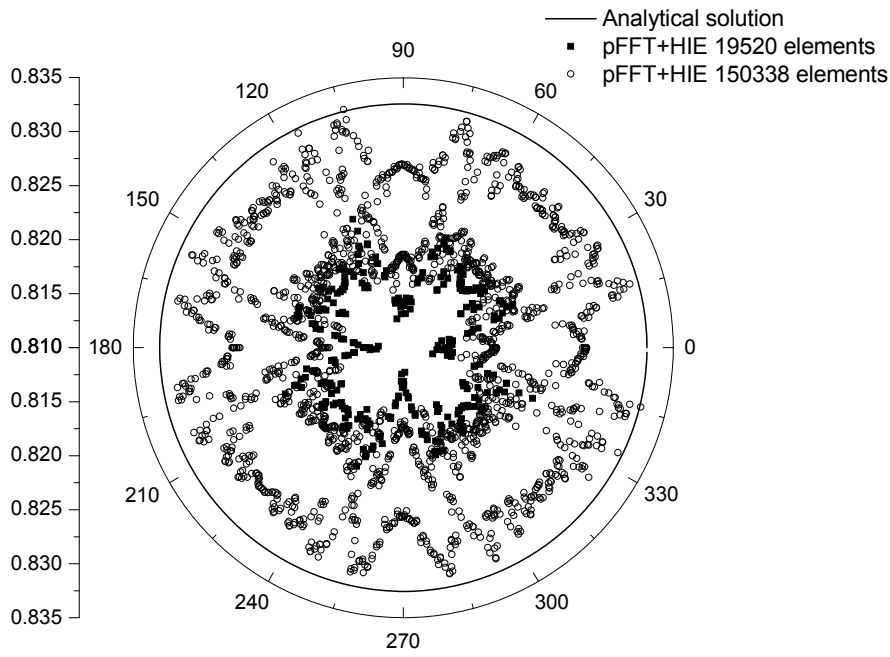

Figure 5: Dimensionless surface acoustic pressures on the circle $x=0$ as a function of $\theta$ at $k a=1.50294$.

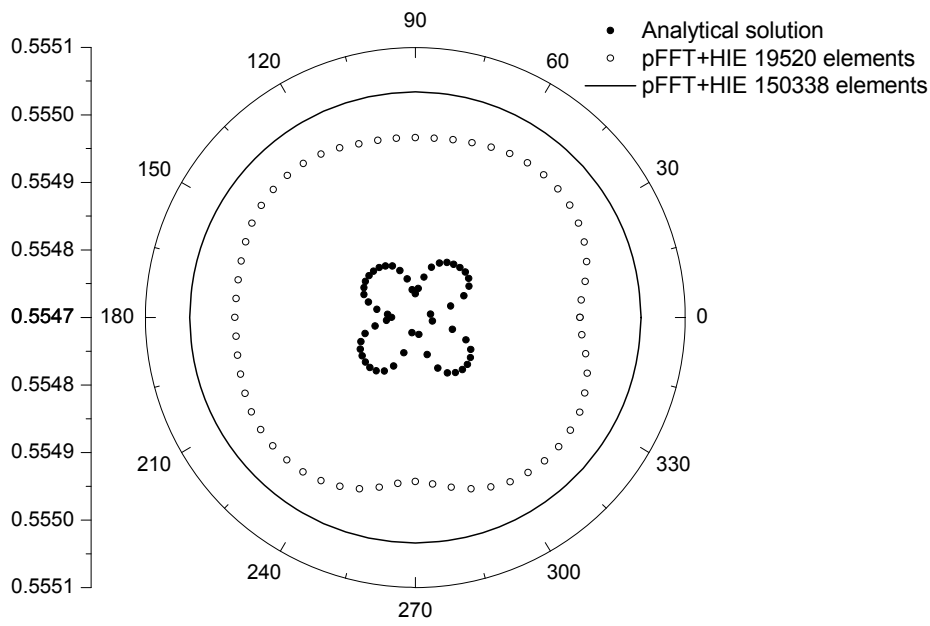

Figure 6: Dimensionless acoustic pressures on the circle $(x=0, r=1.5)$ as a function of $\theta$ at $k a=1.50294$. 
The exterior field solutions can be found using the direct method based on the numerical solutions found on the structural surface. Comparison between the dimensionless acoustic pressures on the circle $(x=0, r=1.5)$ as $k a=1.50294$ computed by the pFFT accelerated Helmholtz integral equation (HIE) and the corresponding analytical solutions is presented in Figure 6. Similarly, two discretizations with 19520 and 1503384 triangular elements are employed. The $L_{2}$-norm errors between the numerical solutions found by the pFFT accelerated $\mathrm{HIE}$ and the corresponding analytical solutions are respectively $0.049 \%$ and $0.011 \%$ for the discretizations with 19520 and 150338 triangular elements.

\section{Conclusions}

A 3-D fast BEM based on the precorrected-FFT accelerated Helmholtz integral equation is developed for the acoustic radiation problems. The computational cost, consumed memory and accuracy of this method are demonstrated through the simulation of a pulsating sphere acoustic radiation. The same as those found in the acoustic scattering problems, the computational costs of the precorrectedFFT accelerated Helmholtz integral equation oscillate along the line $O(n \log n)$ slightly. The numerical results agree well with the corresponding analytical solutions both on the surface and in the exterior domain.

\section{Acknowledgements}

This project was supported by NSFC (11172132); open fund of State Key Laboratory of Structural Analysis for Industrial Equipment (GZ1105); the Priority Academic Program Development of Jiangsu Higher Education Institutions.

\section{References}

[1] Cheng A.H.D. and Cheng D.T., Heritage and early history of the boundary element method. Engineering Analysis with Boundary Elements, 29, pp. 268-302, 2005.

[2] Greengard L. and Rokhlin V., A fast algorithm for particle simulations. Journal of Computational Physics, 73(2), pp. 325-348, 1987.

[3] Wang H.T., Lei T., Huang J.F. and Yao Z.H., A parallel fast multipole accelerated integral equation scheme for 3D Stokes equations. Int. J. Numer. Meth. Engng., 70, pp. 812-839, 2007.

[4] Sanz J.A., Bonnet M. and Dominguez J., Fast multipole method applied to 3-D frequency domain elastodynamics. Engineering Analysis with Boundary Elements, 32, pp. 787-795, 2008.

[5] Zheng C.J., Matsumoto T., Takahashi T. and Chen H.B., A wideband fast multipole boundary element method for three dimensional acoustic shape sensitivity analysis based on direct differentiation method. Engineering Analysis with Boundary Elements. 36(3), pp. 361-371, 2012. 
[6] Phillips J.R. and White J.K., A precorrected-FFT method for electrostatic analysis of complicated 3-D structures. IEEE Transactions on ComputerAided Design of Integrated Circuits and Systems, 16(10), pp. 1059-1072, 1997.

[7] Newman J.N. and Lee C.H., Boundary-element methods in offshore structure analysis. Journal of Offshore Mechanics and Arctic Engineering, 124(2), pp. 81-89, 2002.

[8] Ding J. and Ye W., A fast integral approach for drag force calculation due to oscillatory slip Stokes flows. International Journal for Numerical Methods in Engineering, 60(9), pp. 1535-1567, 2004.

[9] Yan Z.Y., Zhang J., Ye W. and Yu T.X., Numerical characterization of porous solids and performance evaluation of theoretical models via the precorrected-FFT accelerated BEM. CMES: Computer Modeling in Engineering and Sciences, 55(1), pp. 33-60, 2010.

[10] Yan Z.Y., Simulation of acoustic scattering by the fast BEM approach. Journal of Hydrodynamics, 22(5), pp. supplement: 948-953, 2010.

[11] Xiao J.Y., Ye W., Cai Y.X. and Zhang J., Precorrected FFT accelerated BEM for large-scale transient elastodynamic analysis using frequencydomain approach. Int J Numer Methods Eng, 90(1), pp. 116-134, 2012.

[12] Yan Z.Y. and Gao X.W., The development of the pFFT accelerated BEM for 3-D acoustic scattering problems based on the Burton and Miller's integral formulation. Engineering Analysis with Boundary Elements, 37, pp. 409-418, 2013.

[13] Yan H. and Liu Y., An efficient high-order boundary element method for nonlinear wave-wave and wave-body interactions. Journal of Computational Physics, 230, pp. 402-424, 2011.

[14] Jiang S.C, Teng B., Gou Y. and Ning D.Z., A precorrected-FFT higherorder boundary element method for wave-body problems. Engineering Analysis with Boundary Elements, 36, pp. 404-415, 2012.

[15] Yan Z.Y., Zhang J. and Ye W., Rapid solution of 3-D oscillatory elastodynamics using the pFFT accelerated BEM. Engineering Analysis with Boundary Elements, 34(11): Sp. Iss. SI, pp. 956-962, 2010.

[16] Altman M.D., Bardhan J.P. and White J.K., FFTSVD: A fast multiscale boundary-element method solver suitable for bio-mems and biomolecule simulation. IEEE Transactions on Computer-Aided Design of Integrated Circuits and Systems, 25(2), pp. 274-284, 2006.

[17] Saad Y. and Schultz M., GMRES: A generalized minimal residual algorithm for solving symmetric linear systems. SIAM Journal on Scientific and Statistical Computing. 7, pp. 856-869, 1986.

[18] Stefan Kurz and Sergej Rjasanow, The adaptive cross-approximation technique for the 3-D boundary-element method. IEEE Transactions on Magnetics, 38(2), pp. 421-424, 2002.

[19] Tanaka M., Sladek V. and Sladek J., Regularization techniques applied to boundary element methods. ASME Appl. Mech. Rev., 47(10), pp. 457-499, 1994. 
[20] Chen J.T. and Hong K.H., Review of dual boundary element methods with emphasis on hypersingular integrals and divergent series. Appl. Mech. Rev., 52(1), pp. 17-33, 1999.

[21] Yan Z.Y., Hung K.C. and Zheng H., Solving the hypersingular boundary integral equation in three dimensional acoustics using a regularization relationship. J. Acoust. Soc. Am., 113, pp. 2674-2683, 2003.

[22] Yan Z.Y., Cui F.S. and Hung K.C., Investigation on the normal derivative equation of Helmholtz integral equation in acoustics. CMES: Computer Modeling in Engineering and Sciences, 7(1), pp. 97-106, 2005.

[23] Gao X.W., Yang K. and Wang J., An adaptive element subdivision technique for evaluation of various 2D singular boundary integrals. Engineering Analysis with Boundary Elements, 32, pp. 692-696, 2008.

[24] Yan Z.Y. and Zhang Q., Investigation on the singularities of some singular integrals. CMES: Computer Modeling in Engineering and Sciences, 75(3), pp. 205-222, 2011.

[25] Yan Z.Y., Treatment of sharp edges and corners in the acoustic boundary element method under Neumann boundary condition. CMES: Computer Modeling in Engineering and Sciences, 13(2), pp. 81-90, 2006.

[26] Hirosawa K., Ishizuka T. and Fujiwara K., A simple method avoiding nonuniqueness in the boundary element method for acoustic scattering problem. J. Acoust. Soc. Am., 125(5), pp. 2838-2846, 2009.

[27] Chandrasekhar B., Error analysis of various basis functions used in BEM solution of acoustic scattering. CMES: Computer Modeling in Engineering and Sciences, 56(3), pp. 211-229, 2010.

[28] Burton A.J. and Miller G.F., The application of integral equation methods to the numerical solution of some exterior boundary value problems. Proc. R. Soc. London Ser, A323, pp. 201-210, 1971.

[29] Lachat J.C. and Watson J.O., Effective numerical treatment of boundary integral equations. Int. J. Num. Methods Eng., 10, pp. 991-1005, 1976. 